\title{
ACOUSTO-ULTRASONIC MONITORING OF GLUELINE CURING. PART II. GEL AND CURE TIME
}

\author{
Frank C. Beall ${ }^{1}$ \\ Senior Scientific Specialist \\ Weyerhaeuser Company \\ Tacoma, WA 98477
}

(Received March 1988)

\section{ABSTRACT}

\begin{abstract}
Techniques reported in a previous paper, AU transmission through a $25-$ by $25-\mathrm{mm}$ bond area, were applied to previous and new data to develop a more quantifiable approach to adhesive curing. An increase in half-time to cure with increased bond thickness was related to a corresponding increase in gel time, defined as $t_{0.1}$, the time for one-tenth the change in ultrasonic transmission. Cure time $\left(t_{0 . s}\right.$ $-\mathfrak{t}_{0.1}$ ) was invariant with bond thickness. The effects of water and acetone additives to 30 -min epoxy were analyzed using gel and cure time as a measure of curing acceleration or retardation. Acetone, a known retarder, affected both gelling and curing and had a greater effect than water at the same molar concentration.
\end{abstract}

Keywords: Acousto-ultrasonics, ultrasonics, epoxy, glueline thickness, bonding.

\section{INTRODUCTION}

In Part I (Beall 1987), it was shown that acousto-ultrasonic (AU) transmission can be used to monitor the progress of curing of epoxy adhesives within a glueline. The cure time was arbitrarily chosen as the half-time (the time to reach one-half of the total change in ultrasonic transmission), which related directly to the manufacturer's specified cure time. One unexpected result in the study was an increase in half-time with increased bond thickness from 0.05 to $0.5 \mathrm{~mm}$ (controlled by shims).

The specific objectives of Part II were to determine: 1) the cause of the thickness effect on half-time, and 2) the sensitivity of AU to the influence of adhesive additives.

\section{MATERIALS AND EQUIPMENT}

\section{Adhesive}

A commercial 30-min epoxy (Devcon S31) was used for experimental tests on additives. This adhesive, which was also used in the previous study, had a 30min set time, could be handled in $2 \mathrm{~h}$, and developed full strength in $8 \mathrm{~h}$ (all at room temperature and based on the manufacturer's information). The hardener was a proprietary polyamine. Data reanalyzed from the primary study was based on an epoxy (Devcon S208) having a 5-min set time, 15-min handling time, and full strength cure in $1 \mathrm{~h}$.

\section{Adhesive additives}

Two additives, acetone and water, were blended in the 30 -min epoxy. The maximum concentration for water was $3.5 \%$, the apparent limit of solubility. The

\footnotetext{
${ }^{1}$ Current address: Forest Products Laboratory, University of California at Berkeley, 1301 South 46th St., Richmond, CA 94804.

Wood and Fiber Science, 21(3), 1989, pp. 231-238

(C) 1989 by the Society of Wood Science and Technology
} 
upper limit of acetone was established at about 10\% (by mass) to equal the molar concentration for water at 3.5\%. Acetone was used because of its known retardation effect on the epoxide reaction (Potter 1970). Retardation apparently occurs because acetone is a hydrogen acceptor. Conversely, water accelerates the curing of epoxide/amine systems since it is a hydrogen donor.

\section{Adherends}

The configuration selected for monitoring glueline curing was the same as in the previous paper. The lapped specimens were $3.2 \times 25 \times 115 \mathrm{~mm}$, with a lap length of $25 \mathrm{~mm}$, corresponding to BS 1204, synthetic resin adhesives (phenolic and aminoplastic) for wood. This bonding arrangement was selected primarily because of the ease of ultrasonic monitoring, since each lap-half functions as an ultrasonic electrode and waveguide. Also, the bond area is easily prepared and clamped. The electrodes were hard maple (Acer saccharum Marsh.) with grain angle along the length. The side in contact with the bond had been planed and was freshly sanded before the test.

\section{Bonding fixture}

A fixture was used to orient the electrodes to obtain a $25-\mathrm{mm}$ lap length (Beall 1987). For the current study, the fixture was modified to orient the adherends horizontally, providing better control over adhesive spread. Pressure control was maintained using a $27 \mathrm{~N}\left(6 \mathrm{lb}_{\mathrm{f}}\right)$ constant-force clamp. Transducers were bonded to electrodes with a low temperature hot-melt adhesive, using a special fixture to obtain consistent sensor positioning.

\section{Acousto-ultrasonic system}

The AU system (described in Beall 1987) consisted of a Metrotek pulser operating at $700-\mathrm{Hz}$ repetition rate, delivering a single pulse of very short rise time and width at about 30 -volt peak-to-peak amplitude to the piezoelectric transmitter. The crystal resonated with a peak at $175 \mathrm{kHz}$, but actually provided a rather broad-band output. The ultrasonic energy moved through the waveguide (the maple adherend), glueline, and receiving waveguide to a $75-\mathrm{kHz}$ sensor. By using a lower frequency receiver, the system was "detuned," improving the range of linear signal output. The output of the receiver was filtered (45 to $90 \mathrm{kHz}$ ), amplified, and a pseudo-RMS voltage was generated from the envelope of the output. Both the amplifier and RMS outputs were monitored during curing.

\section{PROCEDURE}

In a typical test, sensors were bonded to the waveguides, a $0.125-\mathrm{mm}$ wire shim attached to the lap area, adhesive added, and the lap clamped. The system gain was typically $200 \times$ (preamplifier, $100 \times$; amplifier, $2 \times$ ). Amplifier output was monitored with an oscilloscope to assure consistency in pulse shape run-to-run. RMS output was displayed in real time to obtain the change in ultrasonic transmission; at the end of the run, this display was dumped to a printer. Repetition rate and system gain were selected to provide the highest gain without pulse interaction and amplifier saturation, respectively.

The resin mix and assembly times were controlled at 30 and $40 \mathrm{sec}$, respectively, to minimize differences in apparent cure time. Additives for the epoxy were 


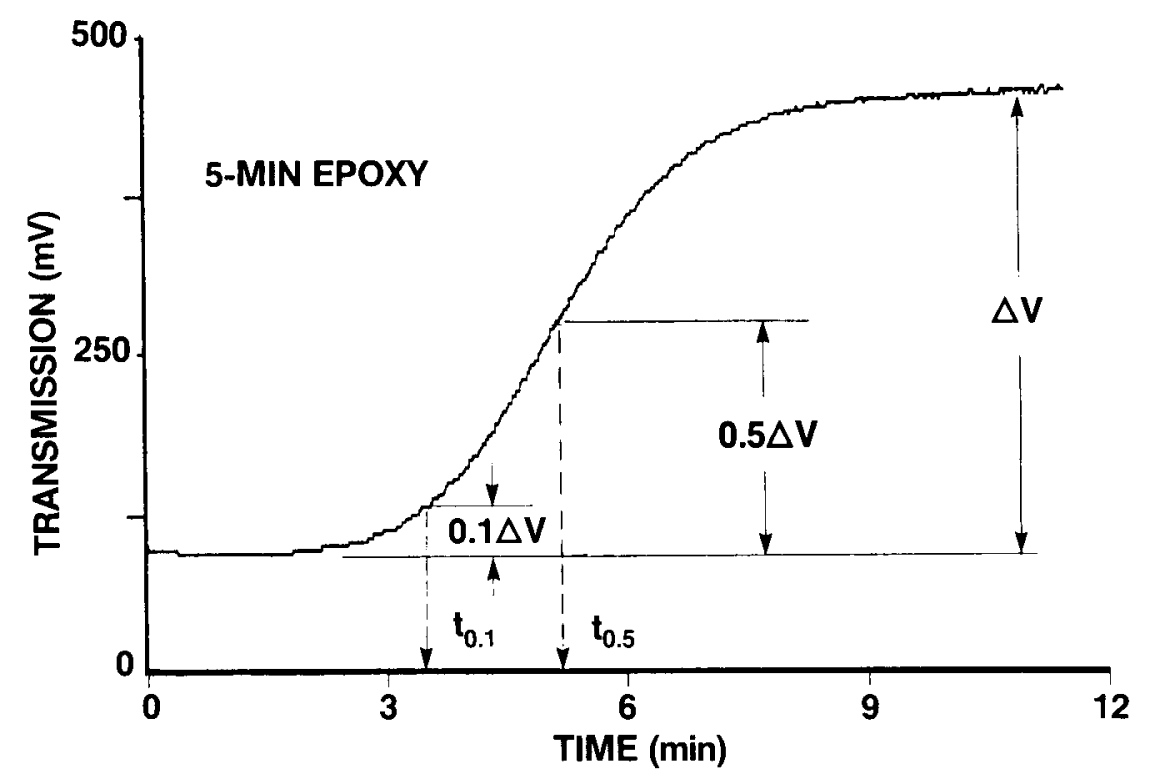

FIG. 1. Procedure for determination of gel time $\left(t_{0,1}\right)$ and half-time $\left(t_{0.5}\right)$ for epoxy adhesive curing.

controlled at 1.7 and $3.5 \%$ (by mass) of water and $3.3,5$, and $10 \%$ (by mass) of acetone.

The change in viscosity with time was analyzed for bulk resin (5-min epoxy, Devcon S208) using a Brookfield viscometer (model RVTD with a No. 7 spindle) to serve as a reference point for gel time.

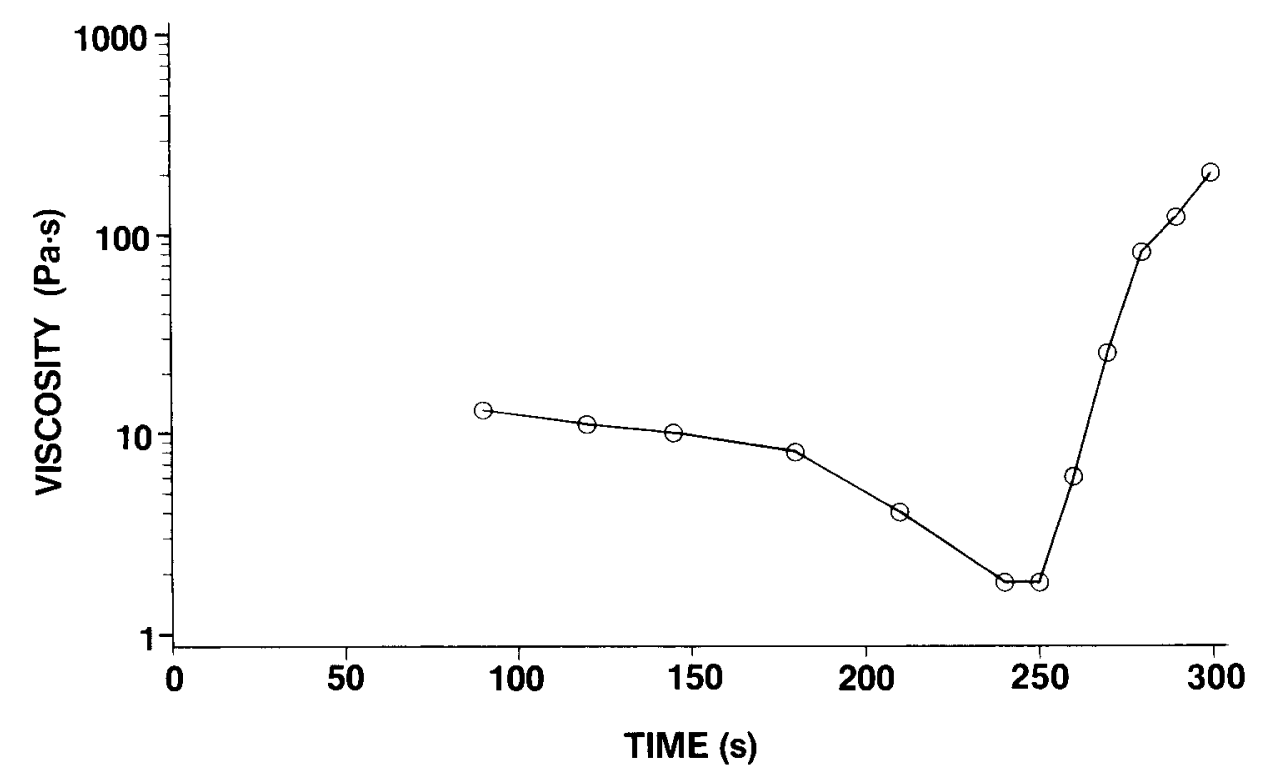

FIG. 2. Increase in bulk viscosity of 5-min epoxy (Devcon S208) as measured in a Brookfield viscometer $(1 \mathrm{~Pa} . \mathrm{s}=1000 \mathrm{cp})$. 


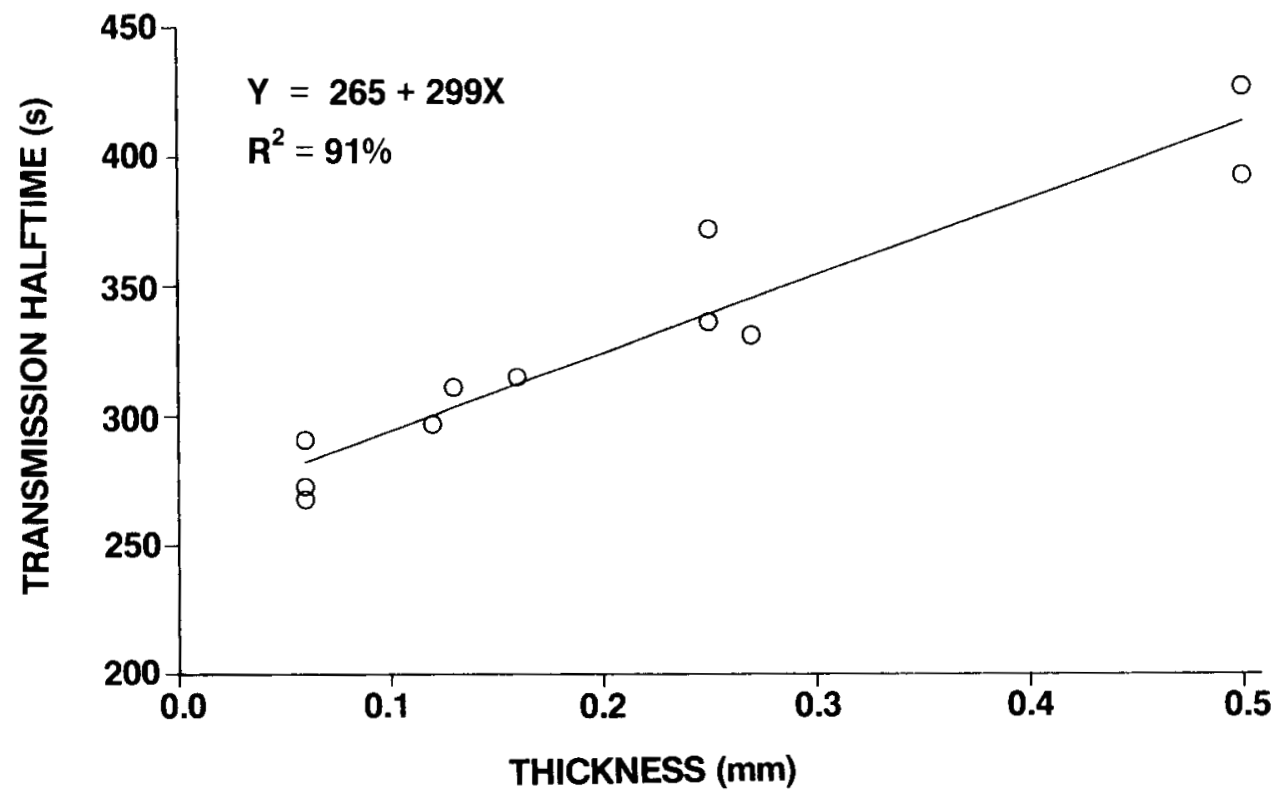

FIG. 3. Effect of glueline thickness on apparent curing time for 5-min epoxy (Beall 1987). Thickness was controlled by addition of wire shims.

\section{RESULTS AND DISCUSSION}

Data analysis

The variables analyzed were half-time $\left(t_{0.5}\right)$, gel time $\left(t_{0.1}\right)$, and cure time $(\Delta t=$ $\left.t_{0.5}-t_{0.1}\right)$. Figure 1 shows how these points were obtained from the transmission

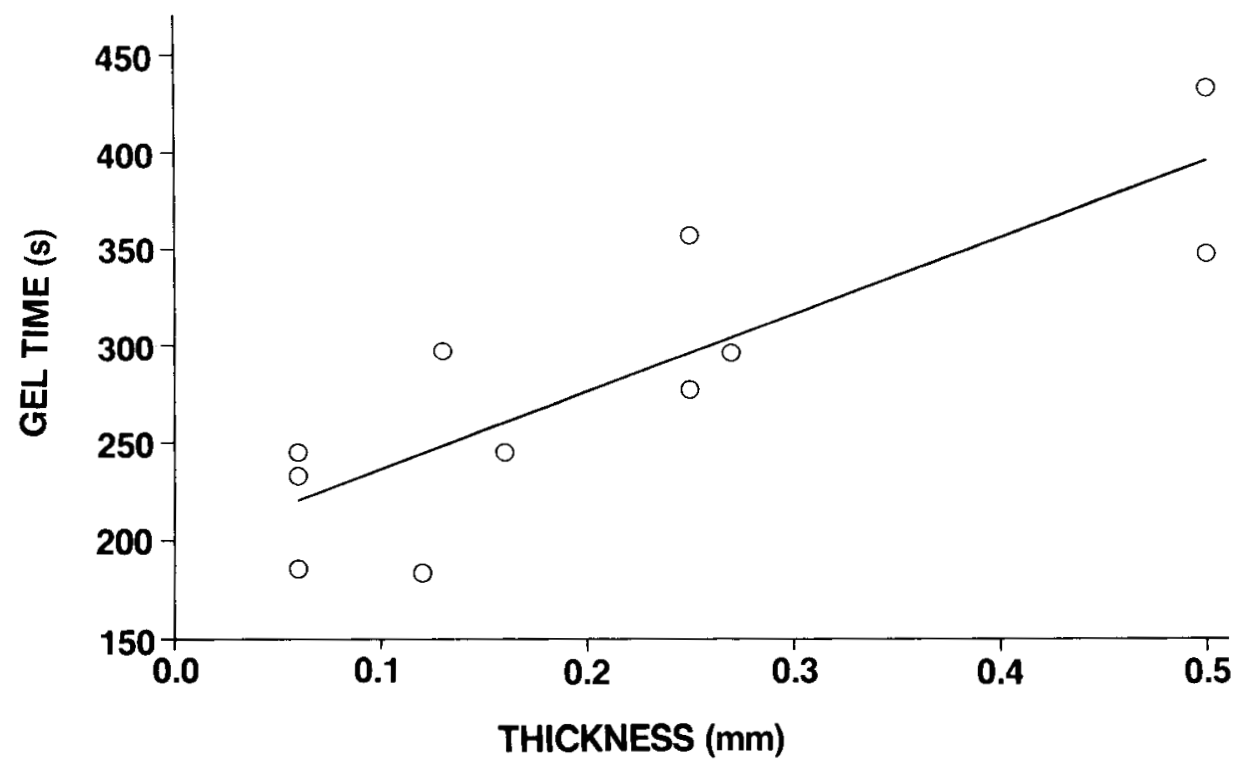

FIG. 4. Relationship of gel time $\left(t_{n .1}\right)$ to bond thickness for 5-min epoxy (Devcon $S 208$ ). 


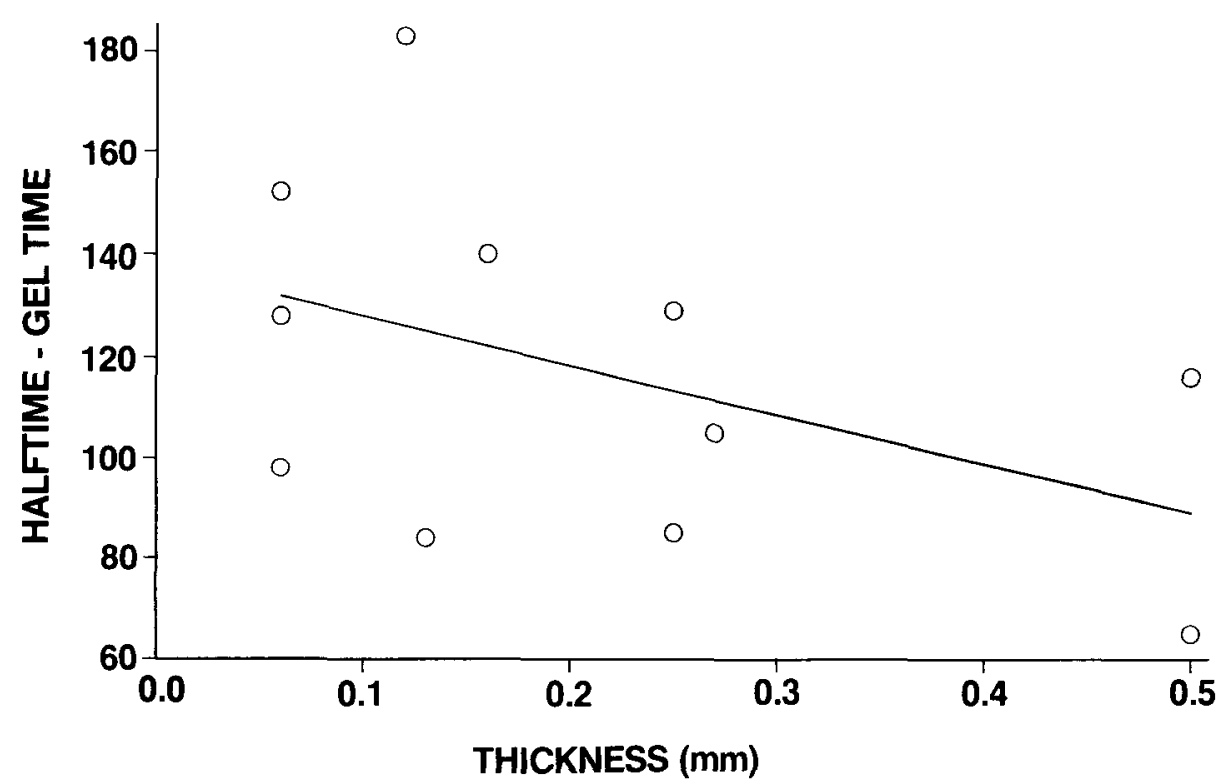

FIG. 5. Relationship of cure time $\left(t_{0.5}-t_{0.1}\right)$ to bond thickness for 5-min epoxy (Devcon $S 208$ ).

curve. The gel time of adhesives is usually defined by an arbitrary increase in viscosity. Since the gel time could not be measured directly within the glueline, an indirect (and arbitrary) estimate was made using the time at which the transmission had reached 0.1 of the total change. In electronics, the 0.1 value is frequently used to define the point of departure of a signal from a baseline.

\section{Gel time of bulk resin}

Figure 2 represents an average for three replicates of the 5-min epoxy from the previous study. The delay in data collection to about $80 \mathrm{~s}$ was the mixing and start-up period. Obviously, the definition of a distinct gel point by absolute or relative viscosity is very arbitrary since the data cannot be easily reduced to a specific value. Also, the generation of exothermic heat would accelerate bulk curing in contrast to the little heat evolved in the lapped bond because of the small adhesive volume and large heat sink of the adherends.

TABLE 1. Mean (and standard deviation) of gel and cure times for runs containing additives vs. controls. Statistical analysis by Student's t-test.

\begin{tabular}{lrcl}
\hline \multicolumn{1}{c}{ Condition } & No. & Gel time (min) & Cure time (min) \\
\hline Control & 15 & $7.2(1.8)$ & $12.8(2.0)$ \\
Water, $1.7 \%$ & 5 & $7.6(1.8) \mathrm{NS}$ & $13.2(1.1) \mathrm{NS}$ \\
Water, $3.5 \%$ & 5 & $9.2(2.3) \mathrm{NS}$ & $16.4(3.3)^{*}$ \\
Acetone, $3.3 \%$ & 5 & $10.0(2.4)^{*}$ & $15.3(1.8)^{* * *}$ \\
Acetone, $5 \%$ & 5 & $8.6(2.0) \mathrm{NS}$ & $18.9(4.3)^{* * *}$ \\
Acetone, $10 \%$ & 5 & $15.4(6.2)^{* * *}$ & $23.6(5.4)^{* * *}$ \\
\hline Levels of significance. $\left.0.95(*) .99^{* * *}\right) 0.99\left(^{* * *}\right)$
\end{tabular}

Levels of significance: $0.95\left({ }^{*}\right), 0.99\left(^{* *}\right), 0.999\left({ }^{* * *}\right)$ 


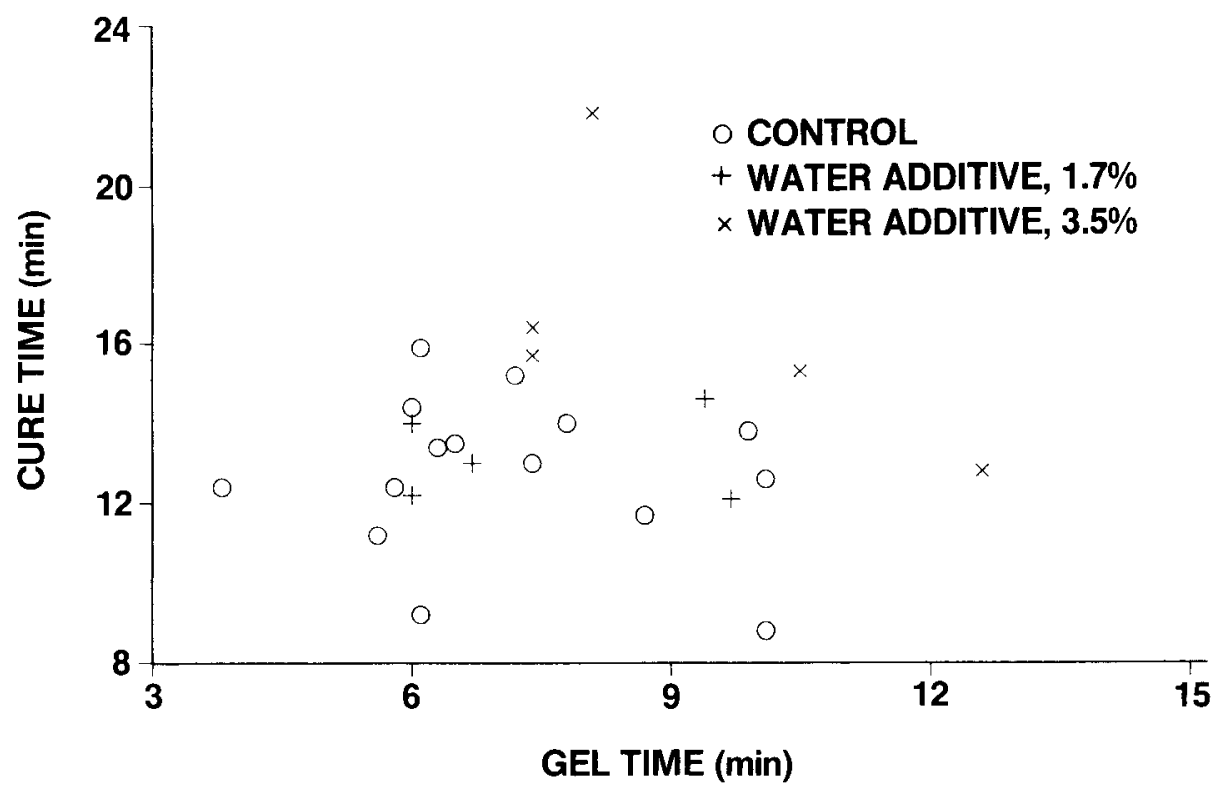

FiG. 6. Effect of water on ultrasonic transmission during curing of 30-min epoxy (Devcon S31).

\section{Effect on bond thickness on curing}

Figure 3 shows the results obtained in the previous paper for 5-min epoxy that clearly indicated an increase in half-time as the bond thickness increased. When gel time data $\left(\mathrm{t}_{0.1}\right)$ were extracted from these data, it was found that gel time also

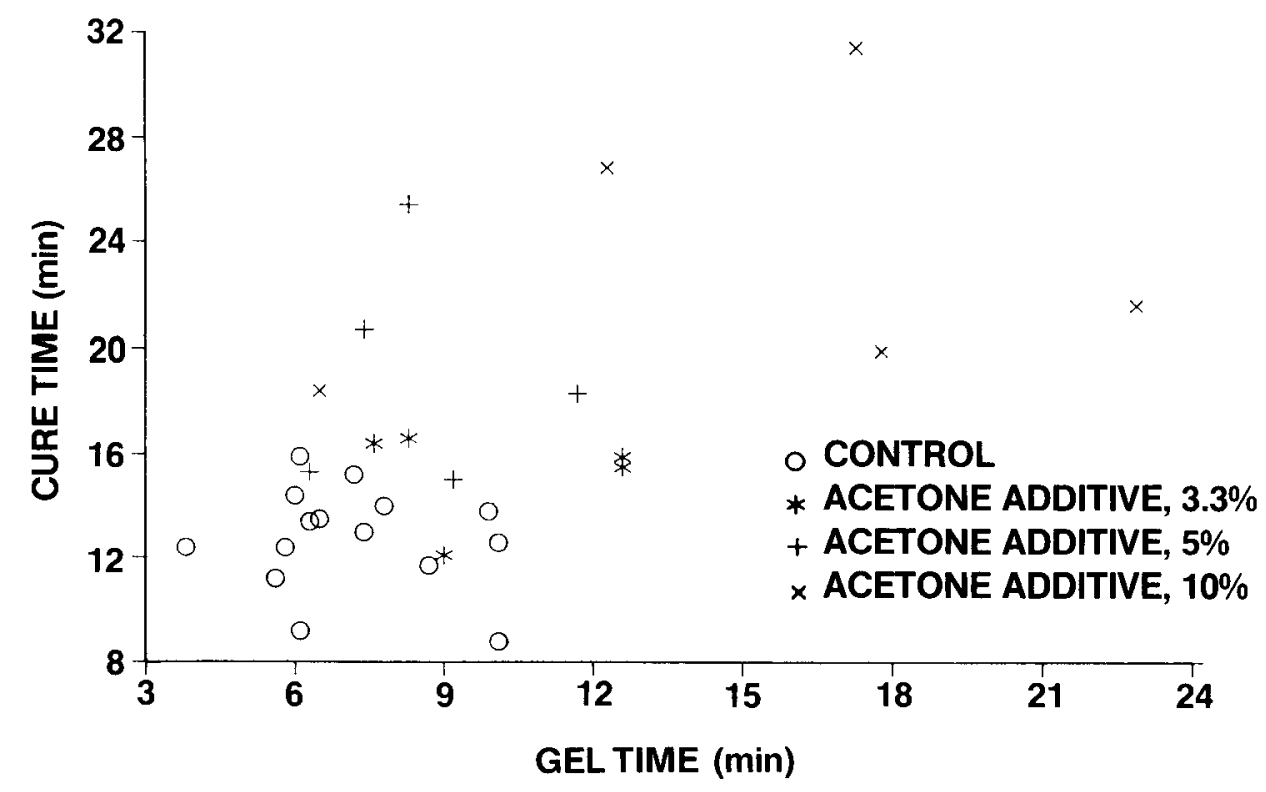

FIG. 7. Effect of acetone on ultrasonic transmission during curing of 30-min epoxy (Devcon S31). 


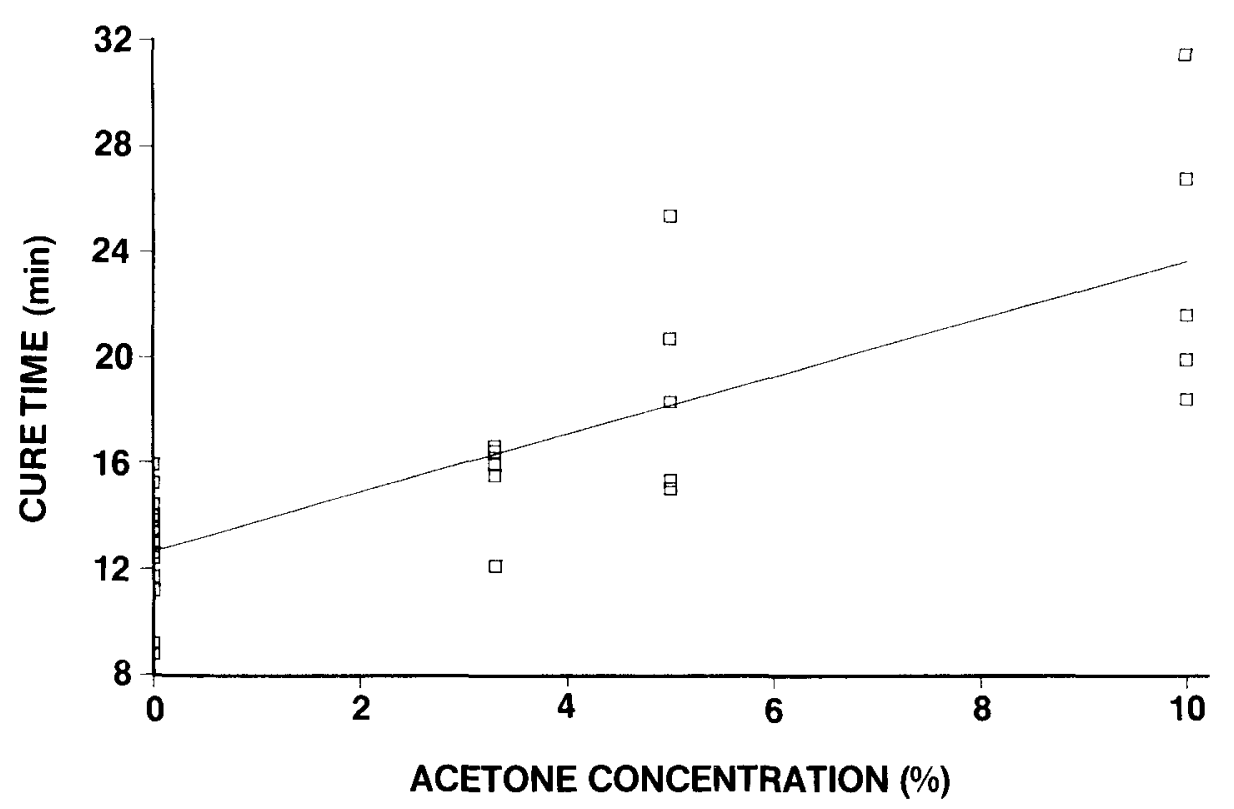

FIG. 8. Effect of acetone concentration on cure time of 30-min epoxy (Devcon S31).

increased with increasing thickness (Fig. 4). (In this data analysis, $70 \mathrm{~s}$, the mixing, spreading, and assembly time was added to both $t_{0.1}$ and $t_{0.5}$, which was not done in the previous paper; this addition simply increases the absolute times of $t_{0.1}$ and $t_{0.5}$ by $70 \mathrm{~s}$, without affecting $\Delta t$ ). Furthermore, if curing time $(\Delta t)$ is compared with bond thickness (Fig. 5), the relationship is essentially random $\left(R^{2}\right.$ $=21 \%$ ). By subtracting gel time from half-time, it becomes possible to eliminate variables such as procedural differences, mixing, variation in thickness both within the bond area and between runs, and reaction differences. Also, the determination of gel time within the actual bond area can provide insight into the influence of the substrate and the adhesive on the overall curing process. The results shown in Figs. 4 and 5 suggest that the adherend surface acts as an accelerator for adhesive gelling, but not adhesive curing. The advantages of the calculated gel time $\left(t_{0.1}\right)$ over that determined by bulk viscosity (Fig. 2) are repeatability, ease of quantification, and lack of an exothermic acceleration. Furthermore, the exothermic heat from the bulk viscosity technique accelerates the cure implying that an increased bond thickness would also reduce the cure time. However, cure time increases with bond thickness, indicating that bulk techniques can provide misleading gel time data for the curing of film-like bonds.

\section{Effect of additives to epoxy}

Since the above analysis presented an opportunity to partition effects related to gelling and curing, water and acetone were individually added to the $30-\mathrm{min}$ epoxy mix. The values for the gel and cure times are summarized in Table 1, together with the statistical significance, and plotted in Figs. 6 and 7. Equal molar concentrations of water and acetone are indicated with the same symbols in the figures. The addition of $3.5 \%$ water caused a significant difference in cure time; 
the gel time difference was very close to the same level of significance, although not statistically significant (Fig. 6). However, when the amount of water was reduced by one-half, there were no significant differences vs. controls. Based on these data, water appears to be a poor hydrogen donor, affecting the parameters only near the limit of solubility. Acetone (Fig. 7) had a much more pronounced effect. The 10\% concentration resulted in gel and cure times that were approximately double that of the controls and was the only concentration that was significantly different at the $0.1 \%$ level for both parameters. The effect of the two lower concentrations on gel time was not conclusive, but both had about the same effect as that of $3.5 \%$ water. All three acetone concentrations had marked effects on delaying cure, and this effect appeared to be proportional to the level of acetone concentration (Fig. 8). The coefficient of variability of all of the data generally increased as concentrations of additives were increased.

\section{CONCLUSIONS}

1. Gel time for epoxy adhesive within a bond area can be approximated by selecting an arbitrary point of departure of the ultrasonic transmission curve during curing, such as $t_{0.1}$, the time for one-tenth the change in ultrasonic transmission. Gel time $\left(t_{0.1}\right)$ increased with increasing bond thickness for 5 min epoxy (Devcon S208), accounting for an apparent increase in curing time with increasing bond thickness.

2. Cure time can be determined independently of factors affecting gel time by subtracting gel time $\left(t_{0.1}\right)$ from half-time $\left(t_{0.5}\right)$.

3. Water added to epoxy resin can retard curing time of 30-min epoxy (Devcon S208), but has little or no effect on gel time.

4. Acetone affects both gel and cure time of 30-min epoxy (Devcon S208), having a greater retardation effect on epoxy curing than water at the same molar concentration.

\section{ACKNOWLEDGMENTS}

This study is funded by the United States Department of Agriculture in the 1985 Forestry Competitive Grants Program under the subprogram of Improved Utilization of Wood and Wood Fiber. The author would like to acknowledge the assistance of Mr. Richard H. Young, Jr., who performed the experimental runs.

\section{REFERENCES}

BeALl. F. C. 1987. Acousto-ultrasonic monitoring of glueline curing. Wood Fiber Sci. 19(2):204214.

PotTER, W. G. 1970. Epoxide resins. Springer-Verlag, New York. 